\title{
Frontières
}

\section{La médecine moderne}

\section{Entre Hippocrate et Esculape}

\section{Patrick Vinay, Michelle Dallaire, Serge Daneault, Tom Hutchinson et Balfour M. Mount}

Volume 17, numéro 2, printemps 2005

Surtout, ne pas souffrir

URI : https://id.erudit.org/iderudit/1073496ar

DOI : https://doi.org/10.7202/1073496ar

Aller au sommaire du numéro

Éditeur(s)

Université du Québec à Montréal

ISSN

1180-3479 (imprimé)

1916-0976 (numérique)

Découvrir la revue

Citer ce document

Vinay, P., Dallaire, M., Daneault, S., Hutchinson, T. \& Mount, B. M. (2005). La médecine moderne : entre Hippocrate et Esculape. Frontières, 17(2), 92-95.

https://doi.org/10.7202/1073496ar d'utilisation que vous pouvez consulter en ligne.

https://apropos.erudit.org/fr/usagers/politique-dutilisation/ 


\section{LA MÉDECINE MODERNE ENTRE HIPPOCRATE ET ESCULAPE}

\author{
Dr Patrick Vinay, \\ Faculté de médecine, Université de Montréal. \\ Dre Michelle Dallaire, \\ Faculté de médecine, Université de Montréal. \\ Dr Serge Daneault, \\ Faculté de médecine, Université de Montréal. \\ Dr Tom Hutchinson, \\ Faculté de médecine, Université McGill. \\ Dr Balfour M. Mount, \\ Faculté de médecine, Université McGill.
}

Les concepts présentés dans cet article ont été largement développés par Michael Kearney (Kearney, 2000). Leur importance pour la médecine d'aujourd'hui tire sa sève dans les racines de la médecine grecque. Ils appellent à une transformation de la relation médecin-malade afin d'implanter un nouveau concept de guérison qui s'applique jusque dans les lits de soins palliatifs. Leur importance justifie cette présentation en langue française. Une pleine compréhension de la pensée et des intuitions présentées ici requiert donc d'aller puiser aux travaux de M. Kearney cités dans cet article.

\section{L'HÉRITAGE D'HIPPOCRATE}

Notre héritage médical remonte aux Grecs. Le monde antique vivait avec le sentiment omniprésent de la précarité de la vie. L'importante mortalité infantile, périnatale ou obstétricale, les épidémies, les guerres et une espérance de vie relativement courte rehaussaient pour chacun le miracle d'être en vie et toute la valeur de la santé fragile. La maladie, la douleur et la souffrance fondaient sur chacun sans crier gare et l'on se demandait pourquoi les dieux envoyaient sur les vivants des événements favorables ou défavorables. En contrepartie, de nombreuses approches «thérapeutiques» coexistaient ${ }^{1}$, disparates et souvent fantaisistes, qui n'avaient d'égal que le grand désordre de la maladie. Dans l'imaginaire grec, les morts eux-mêmes continuaient volontiers d'errer sur la terre des vivants. Une douce tristesse semble donc régner sur ce monde antique où plusieurs des plus grandes réalisations humaines sont adossées à l'omniprésence de la mort et au caractère mystérieux du destin, ultime justicier et grand égalisateur.

C'est dans ce contexte qu'apparaît Hippocrate de Cos (né en 460 av. J.-C.). Contemporain de Socrate et de Platon, ce grand médecin se fait remarquer par son approche systématique de la maladie, par ses qualités d'éthique professionnelle et par son dévouement personnel remarqué entre autres lors de la peste d'Athènes (Meyniac, 2004; Mossé, 2004; Remacle, 2004). Son approche tente de déchiffrer, par l'histoire et l'examen minutieux de chaque malade, un tout intelligible permettant d'expliquer les diverses manifestations d'un désordre aux multiples facettes (Berle, 1993, p. 387-392). Hippocrate tente de dresser des liens entre l'environnement, la nutrition et la maladie, de découvrir une "physiopathologie» qui explique les symptômes et qui permette d'implanter ensuite une intervention planifiée donnant une réponse prévisible (Dufresne, 2004). C'est la «logique de la vie» qui est déjà à l'œuvre, l'intuition d'où naîtra toute la médecine scientifique moderne.

Le paradigme hippocratique veut que si on corrige la cause, on fasse disparaître la maladie et la souffrance. C'est une approche logique, rationnelle, qui accepte que l'inconnu demeure, mais qui construit sur l'expérience gagnée avec chaque malade. C'est un acte de foi en une physiopathologie intelligible. L'intervention médicale hippocratique requiert donc du médecin une objectivité clinique et demande de poser un ou des gestes "extérieurs» au patient, initiatives du médecin savant et expérimenté. On essaie de restaurer la santé antérieure du patient, un opus contra naturam, qui renverse le cours naturel de la maladie et rétablit la santé. Les médecins deviennent de fins observateurs qui enrichissent continuellement leur expertise de leurs expériences pratiques partagées entre eux au moyen de communications et d'écrits (Baillere, 2004; Montfort, 2004).

En ce temps, cette approche préscientifique entrait bien sûr en compétition avec une myriade d'autres pratiques, dont certaines d'ordre magique, qui répondaient à l'anxiété et à l'insécurité des hommes. Mais elle a gagné progressivement de l'importance, probablement à cause de son caractère objectif. Notons que la démarche hippocratique s'adresse plus à la maladie qu'au malade, plus à la douleur qu'à la souffrance et que la foi du médecin comme celle du patient dans l'intelligibilité de la maladie est essentielle à sa survie.

Hippocrate doit quand même composer avec l'ombre des échecs parce que sa science est imparfaite, qu'il ne réussit pas toujours à guérir, qu'il ne peut parfois que soulager ou encourager, et que tout patient finit par mourir. Aujourd'hui, la médecine ignore trop souvent la mort, l'éléphant dans la chambre dont on ne parle pas, contre laquelle on n'a pas de recours. 
portrait de Paul-André

... en ta présence

mon cœur s'est réchauffé

\section{LE PARCOURS DU MALADE JADIS ET AUJOURD'HUI}

On sent bien que le parcours du malade est radicalement différent et en quelque sorte étranger à cette approche rationnelle qu'il peut partager mais qu'il vit autrement dans son monde personnel. Dans bien des cas, le malade ne consulte pas le médecin parce qu'il est malade mais bien plutôt parce qu'il est souffrant (Loeser, 2000). Il essaie d'expliquer ses symptômes par des comportements déficients et s'efforce de les transformer. Mais cela ne marche pas toujours. Surpris par l'intime trahison de son corps entré en dysfonction permanente, il se demande pourquoi cela lui arrive à lui.

Il met alors sa confiance dans la médecine qui propose un retour en arrière, une guérison, souvent au prix de nouvelles souffrances. Cela ne marche pas toujours non plus. Rapidement, il se sent coupable de devenir dépendant des autres, des proches comme des soignants. Si la guérison ne vient pas, il refuse l'idée que la maladie devienne une nouvelle réalité permanente avec laquelle il lui faut vivre désormais. Souvent incapable de surmonter ses difficultés propres ou d'obtenir l'aide dont il a besoin, le malade entre dans un état de désarroi et de ressentiment qui éveille une souffrance intense (Daneault et al., 2004, p. 7-11) et un problème aigu de sens. Cette souffrance n'atteint pas seulement le malade aux prises avec sa maladie mais elle touche aussi les proches du malade et, très souvent, les professionnels qui tentent de le soulager (Daneault et al., 2004, p. 7-11).

Après négation, négociation, colère, dépression (Kübler-Ross, 1970) le malade finit par se résigner. Il a évolué intérieurement et ne demande plus à être guéri, mais seulement soulagé et maintenu dans une qualité de vie qu'il devient vite seul à trouver acceptable, tant les bien portants sont malhabiles à juger des besoins des malades. Certains malades retrouvent une sérénité nouvelle, un sourire radieux, une fragile et précieuse existence conjuguée au présent, même s'ils se savent à la dérive sur un radeau sans gouvernail qui suit son chemin propre. Il n'y a pas de malades cancéreux, cardiaques ou déprimés mais seulement



des personnes qui apprennent courageusement à vivre avec le cancer, une maladie cardiaque, la maladie mentale... Cette évolution intérieure ne fait pas partie du paradigme hippocratique comme nous l'avons décrit. Et pourtant elle nous a été enseignée aussi dans la tradition des Grecs.

\section{L'HÉRITAGE DES DISCIPLES D'ESCULAPE}

Esculape est un demi-dieu d'origine thrace (Bakardjiew, 1969, p. 144-146). Fils d'Apollon (dieu-soleil de la science, des arts et des lois) et de la nymphe grecque Coronis (Berle, 1993, p. 387-392; Toole, 1963, 
p. 387-400; Editor, 1960). Nous avons hérité de plusieurs variantes de son histoire mythique, dont nous ne retenons que quelques éléments ici. Esculape vient au monde par césarienne peu après la mort de sa mère. Il a bien failli mourir lui aussi ! Élevé par le sage centaure Chiron (une exception remarquable parmi les bouillonnants centaures, coléreux, batailleurs et désordonnés), il reçoit de lui un enseignement en médecine. Michael Kearney fait remarquer que le guérisseur Chiron est porteur d'une blessure chronique au pied, infligée par une flèche perdue (Kearney, 2000). Le Centaure maître guérisseur est donc lui-même blessé. Esculape son disciple est blessé lui aussi : orphelin, élevé dans un monde de Centaures, il lui faudra un long cheminement pour se réconcilier avec son destin. La blessure du guérisseur semble ici une préparation personnelle précieuse au métier de soignant ou d'accompagnant des malades (Brescia et Tabuada, 1997, p. 345-347; Kearney, 1996).

L'activité d'Esculape le rend vite célèbre et il devient le patron de tous les guérisseurs. Il sera foudroyé par Zeus, parce qu'Hadès se plaint qu'il lui enlève le pain de la bouche! Il ressuscite alors sous la forme d'un serpent... Il aura plusieurs enfants dont Hugieia, déesse de la santé (hygiène). Pour la petite histoire, on le représente souvent avec son chien et son bâton de marche autour duquel s'enroule un serpent représentant peut-être la maladie apprivoisée... symbole souvent repris comme emblème de la médecine (Schwär, 1985, p. 43-49), non sans de fréquentes confusions avec le caducée (Bohigian, 1997, p. 210-211; Frett, 1994, p. 48-50). C'est celui-ci, et non le bâton d'Esculape au serpent unique, qui est d'ailleurs resté, bien à tort, l'emblème de la médecine.

Dans les temples d'Esculape (Tricha, Cos, Épidaure), on reçoit des malades souffrant de toutes les pathologies (Cilliers et Retief, 2002, p. 60-66). Après un voyage plus ou moins long pour atteindre le temple (Asclepion), ceux-ci sont accueillis par les prêtres (nommés «thérapeutes»). Les prêtres d'Esculape balisent pour chaque patient un parcours mystérieux où, après conversations, rencontres avec d'autres malades, sacrifices, bains, diètes, fumées, ils rencontrent le dieu au cours d'un sommeil «incubateur», le plus souvent à la faveur d'un songe qui les fait évoluer intérieurement (McClenahan, 1977, p. 41-42; Hart, 1965, p. 232-236). Le moment de la rencontre est imprévisible et on peut l'attendre longtemps. La rencontre du dieu a le pouvoir de guérir, mais cela ne survient pas toujours. Fréquemment, le malade est amené à renoncer au retour à l'état antérieur, à accepter un nouvel ordre des choses

NE FAUDRAIT-IL PAS FAVORISER

L'EXPOSITION DE NOS ÉTUDIANTS, DE NOTRE PERSONNEL,

À UN APPRENTISSAGE FORMEL À L'ÉCOUTE ACTIVE?

où sa blessure est dorénavant intégrée à sa réalité. Une nouvelle unité intérieure s'est faite, une "guérison » est survenue, même sans disparition de la maladie. C'est un opus naturae qui ne cherche pas alors un retour en arrière, mais bien un nouvel équilibre et une insertion paisible dans la communauté des souffrants.

Ici, c'est le malade, et non le médecin, qui doit poser un geste intérieur. Ici, on s'adresse plus à la souffrance qu'à la douleur. Ici, le médecin peut accompagner jusqu'à la mort et faciliter cette évolution chez le malade. Clairement, le thérapeute comme le malade, tous deux enracinés dans le mystère de la destinée humaine, cheminent ensemble vers une "guérison» commune (Kellie, 1999, p. 1038; Bailey, 1996, p. 257-263). L'interaction entre les deux personnes s'adresse à chacune d'elles plus qu'à la maladie et cette relation demande une expertise subjective chez l'un qui permette à l'autre d'évoluer. Ceci n'est pas le paradigme d'Hippocrate.

\section{HIPPOCRATE ET ESCULAPE}

Michael Kearney (Kearney, 2000) et Balfour Mount (Mount, 2003, p. 1-2) font justement remarquer qu'au temps des Grecs ces deux traditions n'étaient pas opposées comme on l'a longtemps cru (Berle, 1993; Luce, 2001), mais bien complémentaires. Cette opinion repose sur plusieurs observations: Hippocrate œuvrait dans un "Asclepion», sorte de temple-hôpital du temps, dédié à Esculape (Cilliers et Retief, 2002, p. 60-66); ses élèves étaient connus comme des «asclépiens»; le serment d'Hippocrate fait référence à Esculape: «Je jure par Apollon, par Esculape....». Bien plus, Hippocrate raconte lui-même sa rencontre en songe avec le dieu qui lui dit que «la vérité l'aidera dans sa quête d'une médecine efficace» (Kearney, 2000).

Hippocrate ne rejetait donc pas l'approche des disciples d'Esculape, mais la considérait comme complémentaire à la sienne. Les malades faisaient en quelque sorte les deux démarches en même temps, l'objective et la subjective, conduits par la main dans l'une comme dans l'autre. Cette réflexion est importante car elle suggère que la médecine d'aujourd'hui doit bien être héritière de ces deux traditions. Plus encore, la simultanéité des deux approches émerge comme une condition de toute vraie guérison (Mount et Flanders, 2003, p. 1-2). Cette vision suggère que la complexité de l'homme, corps, esprit et être relationnel, appelle une approche holistique de la guérison tenant spécifiquement compte de toutes ces composantes.

\section{QU'EST-CE QUE LA GUÉRISON?}

Dans notre monde contemporain dominé par une vision mécanique de l'homme, on réfère volontiers au concept hippocratique de guérison, c'est-à-dire à un retour vers un état antérieur à celui qui a été radicalement modifié par la maladie. Pourtant, de telles guérisons se produisent rarement: la maladie ne change-t-elle pas toujours le sujet qui en fait l'expérience? De nombreux malades ne peuvent pas espérer un retour en arrière, car ils sont affligés de conditions dites incurables ou chroniques. Le patient souffrant de plusieurs formes de cancer, du diabète, la personne infectée par le VIH, devront apprendre à vivre avec leur maladie en adoptant un nouveau point de vue compatible avec la poursuite d'un sens. Dans ce contexte, il faut donc opposer à la guérison «mécanique» une autre forme de guérison, axée sur l'évolution intérieure vers un soi transformé. Cette évolution réclame de la part du patient comme de la part du médecin, une humilité nouvelle: l'un et l'autre se doivent d'accepter la condition présente de leur existence et leur impossibilité intrinsèque d'immortalité. Il devient alors possible d'évoluer vers une forme nouvelle de guérison dans laquelle l'humain malade abandonne l'illusion de la perfection et consent à partager avec ses semblables, tous également limités ou malades, une solidarité porteuse de transformation.

Alors la question de la finalité de la vie de l'homme se pose: veut-il faire quelque chose de sa vie éphémère? "Faire quelque chose de sa vie» ne trouve écho que dans les solidarités humaines. Pour ramener aux propos d'Antoine de St-Exupéry (1945), la brique ne découvre pas son sens en ellemême. C'est lorsqu'elle devient une part de la citadelle ou du temple que le sens de son existence est consacré. Quand le malade incurable, comme le soignant qui l'accompagne, peuvent s'avouer lucidement leur impuissance fondamentale à vivre un «retour en arrière», ils peuvent chercher ensemble où et comment poser la brique de leur vie. C'est précisément là que la guérison esculapienne peut survenir. 


\section{FORMÉS À LA MÉDECINE HOLISTIQUE?}

Avons-nous acquis une expertise qui permette d'offrir au malade le support personnel dont il a besoin lorsque la maladie le frappe? Il s'agit ici d'une formation subjective, basée sur les intuitions, sur l'intime compréhension du cheminement de l'autre, sur une interprétation fine de ses signaux non verbaux, sur la création d'un espace entre le thérapeute et le malade où celui-ci pourra se sentir à l'aise, accepté, attendu, écouté. Il s'agit d'une expertise basée sur une maturité d'humanité et qui repose par conséquent sur l'expérience de ses propres blessures. Le guérisseur blessé aura lui-même fait le chemin vers la "guérison »; non pas celle du retour en arrière, mais celle de l'ouverture vers un monde imparfait où la souffrance est inévitable, omniprésente, comme un lien entre les hommes. Saki Santorelli (Santorelli, 1999) suggère qu'une "guérison " personnelle est essentielle à une pratique de la médecine holistique et donc particulièrement à celle des soins palliatifs.

Ces sujets ne sont pas formellement inscrits au curriculum de nos facultés, même si plusieurs professeurs encadrent bien nos étudiants et nos résidents, leur permettant une évolution personnelle précieuse. L'Université McGill a créé à cet effet un groupe de travail sur la guérison (Mount et Flanders, 2003, p. 1-2). Ce groupe examine comment le concept de guérison est pris en compte dans la pratique, l'enseignement et la recherche en médecine, en particulier dans les hôpitaux d'enseignement. Il vise une transformation, un enrichissement de la formation en santé pour y faire une plus grande place à l'aventure de la guérison qui peut être pleinement vécue par le malade. À cette fin, ne faudrait-il pas favoriser l'exposition de nos étudiants, de notre personnel, à un apprentissage formel à l'écoute active, à l'écoute transparente, à la communication interpersonnelle, à une compréhension des effets de nos blessures individuelles sur notre capacité d'interaction avec les autres, à la psychologie du malade chronique, à celle du mourant et à celle de leur famille? Nous avons tous en mémoire quelques mentors qui ont jalonné notre évolution dans ces domaines. Nous avons souvent aussi ressenti une insatisfaction de n'avoir évolué que si peu et de ne pouvoir offrir que si pauvrement. Il faut se poser la question de la place consentie à Esculape dans nos organisations médicales si résolument hippocratiques. En dernière instance l'approche de la douleur ne peut en effet que conduire à celle de la souffrance, et Hippocrate ne peut que conduire à Esculape ${ }^{2}$.

\section{Bibliographie}

BAILEY, J.E. (1996). "Asklepios: ancient hero of medical caring", Ann. Intern. Med., 124 , p. 257-263.

BAILLERE, J.B. (2004). «Hippocrate, œuvres complètes », Bibliothèque intermédicale de Paris, <www.bium.univ-paris5.fr >.

BAKARDJIEW, W. (1969). "Thracian origin of Asclepius, god of healing», Ther Hung, 17(3), p. 144-146.

BERLE, E.J. (1993). «The Asclepian tradition in ancient Greek medicine», Occas. Pap. Med. Hist. Aust., 6, p. 387-392.

BOHIGIAN, G.M. (1997). "The staff and serpent of Asclepius», Mo. Med., 94(5), p. 210-211.

BRESCIA, S.R. et R.G. TABUADA (1997). "Self-healing.», Rev. Hosp. Clin. Fac. Med. Sao Paulo, 52(6), p. 345-347.

CILLIERS, L. et F.P. RETIEF (2002). "The evolution of the hospital from Antiquity to the end of the middle ages", Curationis, 25(4), p. 60-66.

DANEAULT, S., V. LUSSIER, S. MONGEAU, P. PAILlÉ, E. HUDON, D. DION et L. YELLE (2004). "The nature of suffering and its relief in the terminally ill: a qualitative study », J. of Palliative Care, 20(1), p. 7-11.

DUFRESNE, J. (2004). "Hippocrate ou l'ébauche d'une médecine scientifique», L'encyclopédie de l'agora, <http://agora. qc.ca/mof.nst>.

FRETT, P.T. (1994). «Medicine's identity crisis revealed. The Asclepius vs. the Caduceus", Minn. Med., 77(10), p. 48-50.

HART, G.D. (1965). "Asclepius, God of Medicine», Can. Med. Assoc. J., 92, p. 232236.

KEARNEY, M. (1996). Mortally wounded: stories of soul pain, Dublin, Marino Books.

KEARNEY, M. (2000). A place of healing: working with suffering in living and dying, Oxford, Oxford University Press.

KELLIE, S.M. (1999). "Cult of Asclepius», Lancet, 354(9183), p. 1038.

KÜBLER-ROSS, E. (1970). On death and dying, New York, Macmillan.

LOESER, J.D. (2000). «Pain and suffering», Clinical Journal of Pain, 16, p. S2-S6.

LUCE, J.V. (2001). "Greek medicine from Asclepius to Hippocrates", Ir. J. Med. Sci, 170(3), p. 200-202.

MCCLENAHAN, J. L. (1977). «The men on the walls - III: Asclepius», Trans. Stud. Coll. Physicians Phila., 45(1), p. 41-42.

MEYNIAC, J.-P., P. VALÉRIE, P. BORGE, A. BIELMAN et A.-L. REY (2004). «MEMO. Le site de l'Histoire, dossier Antiquité-Grèce antique», <www.memo.fr/article.asp ?>.

MONTFORT, M.-L. (2004). "Collection hippocratique à la BIUM », <www.bium.univparis5.fr/histmed/medica/hipp_vf.htm $>$.

MOSSÉ, C. (2004). "Les lecons d'Hippocrate», Le magazine de l'Histoire, <www. epidaure.com/histoire/hippo.html $>$.
MOUNT, B. et E.M. FLANDERS, (2003). «Healing and palliative care: charting our way forward», Palliative Medicine, 00, p. 1-2.

REMACLE, P. (2004). "Notice sur Hippocrate», <http://remacle.org/bloodwolf/erudits/hippocrate $>$.

SAINT-EXUPÉRY (1945), Citadelle, Paris, Gallimard.

SANTORELLI, S. (1999). Heal Thyself, Bell Tower.

SCHWÄR, T.G. (1985). "The rod or staff of Asclepius", J. Forensic Odonto-stomatol., 3(2), p. 43-49.

TOOLE, H. (1963). «Asclepius. I. Asclepius in history and legend », Surgery, 53, 387-400.

\section{Notes}

1. On peut penser que, comme aujourd'hui, les traditions médicales de toutes les grandes civilisations du temps se côtoyaient dans les grandes cités grecques.

2. Les auteurs désirent remercier leurs patients, leurs collègues et le personnel avec qui ils échangent et travaillent en soins palliatifs pour la qualité des conversations et des gestes qui ont animés ces réflexions suscitées ou partagées et pour les entretiens inspirants. 